\title{
Über die Ursache des osmotischen Druckes und der Ionisation (elektrolytischen Dissoziation).
}

\author{
Von \\ J. Traube. \\ V. Abhandlung.
}

In einer vor kurzem ${ }^{1}$ veröffentlichten Abhandlung über das atomare und molekulare Lösungsvolumen gelangte ich $\mathrm{zu}$ folgendem Satze:

Verwandte (gleichwertige) Ionen üben, gleichgültig mit welchem anderen Ion dieselben in der Lösung enthalten sind, eine gleich starke Anziehung auf das Wasser aus.

Es wurde bemerkt, dals dieser Satz unter gewissen Voraussetzungen noch zu verallgemeinern sei und dann lauten würde:

Jedes Gramm- ̈̈quivalent eines beliebigen Ions übt auf das Lösungsmittel bei gleicher Temperatur eine gleich starke Anziehung aus.

Diese Sätze waren abgeleitet worden aus den Beziehungen des Molekularvolumens der homogenen Stoffe zu dem molekularen Lösungsvolumen ${ }^{2}$ der gelösten Stoffe.

Wo immer bei verwandten Stoffen für das Molekularvolumen Regelmälsigkeiten in den Differenzen der auf einander bezogenen Stoffe gefunden wurden, ergab sich, dal's diese Differenzen dieselben waren als die sehr gleichmälsigen Differenzen der molekularen Lösungsvolumina. Da nun ferner für zahlreiche verwandte

1 J. Traube, Diese Zeitschr. (1895) 8, 68 und Ber. deutsch. chem. Ges. (1894) 27, 3177. - Auf Wunsch des Herm Prof. Kontrausch teile ich zu einer in meiner letzten Abhanding S. 75 enthaltenen Anmerkung berichtigend mit, dal's der so bemerkenswerte Richtungswechsel, wclchen eine Anzahl Kurven der molekularen Lösungsvolumina in grolsen Verdünnungen erfahren, bereits in früheren Abhandlungen ron Kohlizauscr und Haldwachs Gött. Nachr. (Juni 1893) und Wied. Ann. (1893) 50, 118 enthalten war.

2 Es wird für obige Konstante mehrfach der Ausdruck ,scheinbares Molekularvolumen" gebraucht. Da dieser Ausdruck leicht zu Milsverständnissen führen kann, insofern derselbe schon in anderem Sinne gebraucht wird, so erlaube ich mir den Vorschlag, dals man sich doch allgemein jener von mir eingefülıten Bezeichnungsweise bedienen möge.

Z. auorg. Chem, VIII. 
Ionen aus den kleinen und oft negativen Werten der molekularen Lösungsvolumina mit Notwendigkeit eine Kontraktion des Wassers gefolgert werden mufste, so führte jene Gleichheit der Differenzen für feste und gelöste Körper zu dem Schlul's, dal's verwandte Ionen eine gleich starke Anziehung auf das Wasser ausüben.

Es konnte ferner keinem Zweifel unterliegen, dafs die Gesetzmälsigkeiten, welche sich für die Differenzen der molekularen Lïsungsvolumina ergeben hatten, in Bezug auf die Differenzen der Molekularvolumina häufig nur deshalb verdeckt wurden, weil sich hier störende Nebeneinflüsse geltend machten; so vor allem die Aggregation der Moleküle zu gröfseren Komplexen. ${ }^{1}$ Dieser Aggregationsgrad der Moleküle durfte nun für verwandte Stoffe ${ }^{2}$ annähernd als gleich angenommen werden; daher blieb in diesem Falle die Gleichheit der Volumdifferenzen für den festen und gelösten Zustand gewahrt. Für nicht verwandte Ionen war aber sicherlich die Gröl'se der komplexen Moleküle meist eine sehr verschiedene, und es konnte daher aus diesen und anderen Gründen nicht auffallen, dals hier Regelmälsigkeiten nur in den Differenzen für den gelösten Zustand vorhanden waren. Unter Berücksichtigung jener störenden Momente schien es aber, dals eine Verallgemeinerung des erwähnten speziellen Satzes erlaubt sei, d. h. für jedes GrammÄquivalent eines beliebigen Ions eine gleich starke Anziehung auf das Lösungsmittel angenommen werden dürfe.

Dieser verallgemeinerte Satz lälst sich in der That aus meinen bisherigen Versuchsergebnissen noch in ganz anderer Weise ableiten, ja es läfst sich, wenn wir mit Drude und Nernst ${ }^{3}$ gewisse Voraussetzungen machen, sogar die Grölse der Kontraktion pro Gramm-Molekül des gelösten Stoffes berechnen.

Nach meinen früheren Mitteilungen ist das atomare Lösungsvolumen von Natrium und Wasserstoff gleich grofs. Die molekularen Lösungsvolumina der starken Mineralsäuren und Natriumsalze wurden so angenähert gleich grol's gefunden, dal's die minimalen $\mathrm{Ab}$ weichungen nur auf kleine Verschiedenheiten des Ionisationsgrades sowie Fehler des Experiments zurückgeführt werden durften.

In meiner ersten Mitteilung ${ }^{4}$ über das molekulare Lösungsvolumen findet sich nun eine Tabelle, welche $u$. a. auch für

1 Vergl. 1. e.

${ }^{2}$ Vergl. S. 340 der folgenden Arbeit.

${ }^{3}$ Vergl. weiter unten S. 326.

4 J. 'Traube, Ber. deutsch. ehem. Ges. (1892) 20̆, 2528. 
schwache Säuren und deren Natriumsalze die Differenzen der molekularen Lösungsvolumina enthält.

Diese Tabelle soll anf Grund neuerer Daten ${ }^{1}$ vervollständigt und korrigiert hier zum Abdruck gelangen. - Die Konzentrationen, welche auf einander bezogen wurden, sind so gewählt, dafs die Tonisation der Säure höchstens $5 \%$ betrug, während das Natriumsalz $\mathrm{zu}$ mindestens $90 \%$ und meist darüber als ionisiert angesehen werden konnte. Arsensäure und Phosphorsäure wurden mit den eiubasischen Salzen verglichen.

\begin{tabular}{lc|lrr} 
& $\mathrm{H}-\mathrm{Na}$ & & $2(\mathrm{H}-\mathrm{Na})$ \\
Metaborsäure & 12.7 & Malonsäure & 2. & 12.8 \\
Phosphorsäure & 12.4 & Bernsteinsäure & 2. & 12.4 \\
Arsensäure & 13.5 & Maleinsäure & 2. & 11.0 \\
Chloressigsäure & 11.6 & Äpfelsäure & 2. & 13.6 \\
Ameisensäure & 10.3 & Weinsäure & 2. & 10.1 \\
Essigsäure & 12.1 & Citrakonsäure & 2. & 12.5 \\
Propionsäure & 12.7 & Mesakonsäure & 2. & 10.6 \\
Buttersäure & 13.8 & Itakonsäure & 2. & 12.2 \\
Milchsänre & 13.1 & & &
\end{tabular}

Die Differenz $\mathrm{H}-\mathrm{Na}$, bei gleichem Ionisationsgrade von Säure und Salz ist gleich 0, dagegen beim Vergleich nahezu völlig ionisierten Salzes mit nicht ionisierter Säure im Mittel=12.2.

Ich war bisher geneigt, die Abnahme des molekularen Lösungsvolumens, welche mit wachsender Ionisation einer Lösung stets eintritt, auf den Umstand zurückzuführen, dafs die Summe der Ionen ein kleineres Volumen einnimmt als das nichtionisierte Molekül.

Es ist das Verdienst von Drude und Nernst, zuerst die entgegengesetzte Annahme gemacht zu haben. Sie folgern aus der Additivität des Molekularvolumens, dals die Summe der Ionen wahırscheinlich gleich sei dem Volumen der nichtionisierten Moleküle.

Jenes Argument scheint allerdings nicht stichhaltig. Immerhin lälst sich zeigen, ${ }^{2}$ dals bei der Spaltung komplexer in einfache

1 Die Werte sind entnommen den Arbeiten von ReYHer, Zeitschr. pleys. Chem. (1888) 2, 744. Le Buane, Zeitsehr. phys. Chem. (1889) 4, 557. Lauenstein, Zeitschr. phys. Chem. (1892) 9,420. Traube, Ann. Chem. Pharm. (1891) 265, 27 und Diese Zeitsehr. (1892) 3, 11, (1894) 8, 12. Komraugch und Harswachs, Gölt. Nachr. (1893) 350. Kannonikof, Journ. pr. Chem. (Neue Folge, $1885)$ 31, 321.

2 Vergl. weitcr unten S. $\$ 34$. 
Moleküle stets eine Volumvergrölserung eintritt; dementsprechend würle eine Volumverkleinerung bei dem einigermalsen vergleichbaren Vorgang der Ionisation nicht wahrscheinlich sein. Auch wäre nicht recht einzusehen, weshalb die Volumverkleinerung für die verschiedenartigsten Säureionen gleich grol's sein sollte.

Trotz allem ist jene Annahme von Drude und Nerssm noch recht hypothetisch; es scheint jedoch, dals die Folgerungen derselben Recht geben.

Aus der Annahme, dafs jene Volumkontraktion bei dem Vorgang der Ionisation nicht auf Kosten einer Volumänderung der ionisierten Moleküle zurückzuführen ist, ergiebt sich ohne weiteres die Annahme einer gleich grolsen Kontraktion des Wassers pro Gramm-Äquivalent gelösten Stoffes.

Diese Kontraktion beträgt für jedes Gramm-Äquivalent eines einwertigen Ions im Mittel $=12.2 \mathrm{ccm}$.

Wenn ein Äquivalent einer starken Base mit einem Äquivalent einer starken Säure in wässeriger Lösung neutralisiert werden, so besteht nach den neueren Anschauungen der wesentlichste Vorgang in der Bildung eines nichtionisierten aus einem ionisierten Wassermolekül. Der Vorgang müfste hiernach bei vollständiger Ionisation mit einer Ausdehnung von $2 \times 12.2 \mathrm{ccm}$ pro Gramm- $\ddot{A} q u i v a l e n t$ gebildeten Salzes verbunden sein.

In Wahrheit beträgt nach den neuesten Messungen Tamian's ${ }^{1}$ die Ausdehnung für verdünnteste Lösungen pro Gramm- ̈̈quivalent $=23 \mathrm{ccm}$, eine Zahl, welche der berechneten sehr nahe kommt. ${ }^{2}$

Drude und NeRNst ${ }^{3}$ folgern in einer uns erst ror kurzem bekannt gewordenen Arbeit: Über Elektrostriktion durch freie Ionen, aus theoretischen Betrachtungen, dafs die elektrischen Ladungen der in Wasser gelösten Ionen eines Elektrolyten die Ursache der erheblichen Volumänderungen seien, welche das Lösungsmittel durch den gelösten Stoff erfährt. Sie machen hierbei die Annahme, dal's bei Nichtelektrolyten das Volumen der gelösten Substanz ungefähr so grol's sei, wie dasjenige im freien (flüssigen oder festen) Zustande.

${ }^{1}$ G. Tammax, Zeitschr. phys. Chem. (1895) 16, 96.

${ }^{2}$ Es sei bemerkt, dal's die $138 \mathrm{~K}$ (bei vollständiger Dissoziation $=165 \mathrm{~K}$ ), welche bei der Neutralisation einer starken Säure und Basis frei werden, nicht, wie Arrhentus u. a. annahmen, die Dissoziationswärme des Wassers bezeichnen. $\mathrm{F}_{\mathrm{s}}$ entspricht vielmehr $165 \mathrm{~K}=\mathrm{H}, \mathrm{OH}-\mathrm{H}$, aq-OH, aq.

3 Drone und Nernst, Zeitschr. phys. Chem. (1894) 15, 79. 
Auf diese Annahme hin wird eine Theorie der Elektrostriktion entwickelt.

Diese Theorie kann unmöglich den Thatsachen entsprechen, denn, wie ich weiter unten ${ }^{1}$ zu zeigen gedenke, besteht zwischen Elektrolyten und Nichtelektrolyten in Bezug auf die Kontraktion gar kein Unterschied. Auch wurden von mir in anderen Lösungsmitteln als Wasser oft sehr erhebliche Kontraktionen festgestellt, obwohl man die Zahl der gelösten Ionen in jenen Fällen als nicht sehr grol's ansieht. Die hypothetische elektrische Ladung der gelösten Ionen ist daher sicherlich nicht die Ursache der Kontraktionserscheinungen. Von besonderem Interesse sind dagegen die weiteren Ausführungen der Verfasser.

Unter der bereits erwähnten Annahme, dafs das Volumen der gelösten Moleküle bei dem Vorgang der Ionisation ungeändert bleibt, wird aus einigen Werten von Kohlrausch und HaLuwachs, sowie von mir der Satz gefolgert, „dafs für die Elektrostriktion pro GrammMolekül eines binären, aus zwei einwertigen Ionen bestehenden Elektrolyten sich in einer Anzahl Fälle (etwa bis auf die Beobachtungsfehler) gleiche Zahlen $(8-11 \mathrm{ccm})$ ergeben haben." Wird für Elektrostriktion das Wort Kontraktion gesetzt, so ist dieser Satz im wesentlichen identisch mit dem von mir aufgestellten Satze.

Allerdings stiitzt sich bei Drude und Nernst die Begründung jenes Satzes nur auf die folgenden fünf Zahlen:

Essigsäure $9.5 \mathrm{ccm}$.

Phosphorsäure $11.3 \mathrm{ccm}$ bezw. $12.4 \mathrm{ccm}$.

Monochloressigsäure $10.6 \mathrm{ccm}$.

Weinsäure $8.1 \mathrm{ccm}$.

Davon dürften nur die ersten beiden Zahlen nach einwandfreier Methode erlangt sein. Es wird hier zu dem Wert des ionisierten Natriumacetats bezw. -monophosphats der Wert $\mathrm{HCl}-\mathrm{NaCl}$ addiert, eine Methode, die sich nach S. 324 noch etwas einfacher gestaltet.

Die letzten drei Werte wurden dagegen erhalten, indem die Differenz der molekularen Lösungsvolumina für ein bestimmtes Konzentrationsintervall dividiert wurde durch die Differenz der aus dem Leitvermögen berechneten Dissoziationsgrade.

Ich betrachte die annähernde Übereinstimmung der obigen drei Werte als eine rein zufällige; in einer weit gröfseren Anzahl von Fällen werden durchaus andere Werte erhalten. Es kann dies

1 Vergl. S. 331 ff. 
auch gar nicht anders sein, da die scheinbare Abnahme des molekularen Lösungsvolumens mit abnehmender Konzentration aufser von der Ionisation auch von der Spaltung der komplexen Moleküle ${ }^{1}$ abhängt. Es soll nun versucht werden, die Bedeutung jenes von zwei Seiten übereinstimmend gefolgerten Satzes klarzulegen.

Gegen die Theorien des osmotischen Drucks und der elektrolytischen Dissoziation ist mit Recht wiederholt der Vorwurf erhoben worden, dals bei dem Bestreben, die völlige Analogie des Zustandes in den Gasen und verdünnten Lösungen nachzuweisen, den Lösungsmitteln eine zu passive Rolle angewiesen wurde. Auch auf die Frage, welche Kräfte thätig seien, um die Ionen eines Elektrolyten im Sinne der Dissoziationstheorie von einander zu trennen oder den Ionenverband zu lockern, wurde nie eine genügende Antwort erteilt.

Allerdings halten Arrhensus ${ }^{2}$ und Nernst ${ }^{3}$ die Annahme einer Anziehung von Lösungsmittel und gelöstem Stoff mit der Theorie des osmotischen Druckes sehr wohl für vereinbar, CIAMician ${ }^{4}$ wie auch in ähnlicher Weise WERNER ${ }^{5}$ suchen die Ursache der elektrolytischen Dissoziation in der Wasseranziehung der freien Ionen, aber im grolsen und ganzen wird doch so sehr die Rolle des Lösungsmittels vernachlässigt, dal's gewil's VAN DER $\mathrm{WAALS}^{6}$ ein Recht hat zu behaupten, ,dals man nicht richtig handle, wenn ein Stoff in einem andern gelöst sei, dem Lösungsmittel jede Wirkung auf den aufgelösten Stoff' abzusprechen, was in letzter Zeit doch vielfach geschehen sei." Die Beobachtungen stimmen nach vaN DER WaALS nicht mit der Hypothese der clektrolytischen Dissoziation überein, ,es sei denn, dals man zu gleicher Zeit annimmt, dafs das Lösungsmittel eine starke Wirkung auf den gelösten Stoff ausübt und umgekehrt". Die Wirkung des Wassers auf die Ionen ist nach ihm eine stärkere als auf die Salzmoleküle.

H. A. Lorentz ${ }^{7}$ leitet in einer Abhandlung: Zur Molekulartheorie verdünnter Lösungen, die Gesetze des osmotischen Druckes

1 Vergl. S. $334 \mathrm{ff}$.

${ }^{2}$ Arrhanus, Zeitschr. phys. Chem. (1888) 2, 500.

${ }^{3}$ Nernst, Theoret. Chem. (1893) 226.

${ }^{4}$ Cinmician, Zeitschr. phys. Chem. (1890) 6, 408.

"Wetren, Diese Zeitschr. 3, 267. R. s. Ber. deutsch. clem. Ges. (1893) $\mathbf{2 6}, 353$.

6 vas ber WaAts, Zeilschr. phys. Chem. (1891) \&, 188 u. 215.

i II. A. Lonentz, Zeilsche phys. Chem. (1891) 7, 36. 
ab, indem er die beiden Voraussetzungen macht, dafs: 1. ein Molekül eines gelösten Stoffes im Mittel eine ebenso grol'se kinetische Energie besitzt, wie ein Gasmolekül bei der gleichen Temperatur; 2. der gelöste Stoff seitens des Lösungsmittels eine Anziehung erfährt, die gleich und entgegengesetzt der äufseren Kraft ist, welche auf die verdrängte Menge der Flüssigkeit wirken würde.

HofMeIster ${ }^{1}$ hat in einer Arbeit über die wasserentziehende Wirkung der Salze das Fällungsvermögen der Salze für gewisse Kolloide aus ihren wässerigen Lösungen untersucht. Für Lösungen von Eisenoxyd etc. ergab sich, dals annähernd gleiche Gramm - Molekulargewichte der verschiedensten binären Salze vorhanden sein müssen, damit Fällung erfolgt. Der Grund dieser Erscheinungen wird wohl mit Recht in der Fähigkeit der Salze gesucht, infolge ihrer Anziehung zum Wasser, der Liösung des Kolloids Wasser zu entziehen. Eine gleiche Anzahl verschiedener Ionen würde demnach eine gleiche Anziehung auf das Wasser ausüben.

Endlich hat G. C. SchMIdT ${ }^{2}$ im Anschluls an die Arbeiten von Gone die Grölse der Adsorption festgestellt, welche stattfindet, wenn gleiche äquivalente Mengen enthaltende Salzlösungen mit einer bestimmten Menge feinen Sandes in Berührung gebracht wurden. Es ergab sich, dal's die Adsorption der Salze an der Oberfläche des Sandes für gleiche Äquivalente gleich grols war; ein Ergebnis, welches wohl auch am besten in einer gleich starken Anziehung des Wassers auf die Ionen seine Deutung findet. ${ }^{34}$

${ }^{1}$ Hopmeister. Arch. f. exper. Pathol. u. Pharmakol. (1888) 25.

R. s. Zeitschr. phys. Chem. (1888) 2, 860.

${ }^{2}$ Sснмпт, Zeitsehr. phys, Chem. (1894) 15, 56.

${ }^{3}$ Poisson hat eine von Volkmans und Rothes an Salzlösungen geprüfte Gleichung aufgestellt, welche lautet: $\gamma=u_{1}^{2} \gamma_{1}+2 u_{1} u_{2} \gamma_{12}+u_{2}^{2} \gamma_{2}$. Hier bezeichnet $\gamma$ die Oberflächenspannung der Lösung, $\gamma_{1}$ und $\gamma_{2}$ diejenige von Salz und Wasser, $u_{1}$ und $u_{2}$ bezeichnen das Volumen vorhandenen Wassers und Salzes, und $\gamma_{12}$ ist das Mals der Anziehung zwischen Salz und Wasser. Für dicse Grölse $\gamma_{12}$ berechnet nun Volkmann für die verschiedensten Salze gleiche Werte. Diese Werte sind sogar nicht sehr verschieden von den von uns berechneten Werten, nämlich im Mittel $=10$. Trotzdem handelt es sich hier um keino Übereinstimmung, denn $\gamma_{13}$ bezeichnet nieht die Anziehung pro Gramm-Molekül gelösten Salzes. Vergl. Ostwald, Lehrb. d. allg. Chem. (1891) 1, 536.

${ }^{4}$ Hingewiosen sei auch hier anf die sogenannte Browssche Bowegung, welche, wie kürzlich von Mrade Bache, Proc. Amer. Philos. (1894) 33, 163, nachgewiesen wurde, nur im Wassor eintritt, und daher eine spezifische Wirkung dieses Lösungsmittels darstellt. Da, wie ich foststellen konnte, grölsere Kontraktionen im allgemeinen nur in Wasser cintreten, so möchte ich ammehmen, 
Erinnern wir uns nunmehr an den für die Aufstellung der vaN'T Hofrschen Theorie der Lösungen so fundamentalen Versuch, bei welchem Zuckerlösung und Wasser durch eine halbdurchlässige Membran getrennt sind, so mul's zugegeben werden, dal's die unbefangene Beobachtung des Phänomens uns keineswegs zur Annahme eines von Zucker ausgeübten Druckes geführt haben wïrde.

Wenn die Beobachtung lehrt, dals Wasser so lange in die Zuckerlösung flielst, bis sich in dem Behälter ein bestimmter Druck einstellt, so würde die frei von theoretischer Annahme erfolgende Übersetzung dieses Versuches lauten: die Zuckerlösung bezw. der Zucker äbt eine bestimmte Anziehung auf das Wasser aus $;^{1}$ infolgedessen flielst so lange Wasser zur Zuckerlösung, bis das Gleichgewicht sich einstellt. Die Grölse der Anziehung wird durch die osmotische Druckhöhe gemessen.

H's ist wohl anzunehmen, dals, wenn van'T HoF' jener Erscheinung eine andere Deutung gab, ${ }^{2}$ er hierza wesentlich veranlafst. wurde durch den Wunsch, in die Theorie der Lösungen eine Druckgröfse einzuführen, welche das Analngon bildete zu dem Spannkraftsdruck der Gase. Der unbefangene Beobachter mulste, wie mir scheint, zu derjenigen Auffassung gelangen, welche s. Z. Lo'thali Mexer ${ }^{3}$ iiber das Wesen des osmotischen Druckes ausgesprochen hat.

Wir haben nun den Satz aufgestellt: Jedes gleichwertige Ion übt auf das Lösungsmittel eine gleich starke Anziehung aus. Es scheint mir, dafs in diesem Satze die Gesetze des osmotischen Drucks bereits im Keime enthalten sind.

Wemn die verschiedenen Ionen auf das Lösungswasser die gleiche Anziehung ausüben, so mufs auch ihr osmotischer Druck der gleiche sein. Es müssen ${ }^{4}$ Gesetze gelten, welche denen von Boyrs, GAYLussac und Avogadro analog siud. Wenn cin Elektrolyt in Wasser

dals aneh allein das Wasser eine stärkere Anzichung auf freio suspendierte feste Teilchen, wie Karmin, Kochenille ete, ansiibt. Es bildet sich um jedes feste Partikelchen als Centrum infolge der Anziehung eine Wasserkugel, und aus der gegenseitigen Verschiebung dieser Wasserkugeln gegen cinander resultiert dio bekannte Zickzackbewegung der festen Substanz. Jie Anziehung ist auch die Ursache, dal's suspendierte Teilchen sich so lange schwebend erhalten.

${ }^{1}$ Entsprechend kann man annehmen: das Wasser übt einen bestimmten Druck auf die Zuckerlösung aus.

${ }^{2}$ VAN'T HofF, Zeitschr. phys. Chem. (1890) 5, 174.

${ }^{3}$ Tothar Mever, Znitsrhr. phys. Ohem. (1990) 5, 23.

+ Vergl. die Anschauungen von Joren'\%, Seite 328. 
gelöst wird, so wird dieselbe Anziehung, welche die Erscheinungen des osmotischen Druckes veranlalst, hier eine Lockerung des Ionenverbandes herbeiführen.

Ich habe immer den Standpunkt ${ }^{1}$ eingenommen, dals die Annahme einer vollständigen Trennung der Ionen im Sinne der Dissoziationstheorie keineswegs notwendig sei, und mit gewissen Thatsachen im Widerspruch stehe. Hier möchte ich einmal die Frage aufwerfen, ob nicht die Annahme ausreicht, dafs bei der Auflösung eines $\mathrm{NaCl}$-Moleküls nur ein (wenn auch sehr grofser) Teil der Energiemenge, welche das $\mathrm{Cl}$ und Na-Atom mit einander verbindet, durch die Wasseranziehung überwunden wird. Da die in Gestalt dieser Wasseranziehung vorhandene Energiemenge für alle gleichwertigen Ionen gleich grofs ist, so erklären sich auch vom Standpunkte dieser Ionisationshypothese, wie mir scheint, die osmotischen Erscheinungen, die additiven Erscheinungen der Lösungen, die thermischen $\mathrm{Er}$ scheinungen, insbesondere Dissoziationswärmen etc.

Wenn nun aber obige Anschauungen über das Wesen des osmotischen Druckes richtig sein sollen, so muls der zunächst für die Ionen aufgestellte Satz noch einer Verallgemeinerung fähig sein.

Auch jedes Molekül eines nichtionisierten Stoffes mul's alsdann eine gleiche, einer Kontraktion von $12.2 \mathrm{ccm}$ entsprechende Anziehung auf das Wasser ausüben.

Prüfen wir diesen den Anschauungen von Drude und Nersst direkt widersprechenden Satz auf seine Richtigkeit.

Ein grofses mir zu Gebote stehendes Beobachtungsmaterial, dessen Veröffentlichung in nächster Zeit bevorsteht, zeigt, dals auch für die aus wässerigen Lösungen organischer Stoffe berechneten molekularen Lösungsvolumina sich sehr gleichmälsige Differenzen berechnen. Auch hier findet man für die verdünnten Lösungen Gesetzmälsigkeiten, da, wo für die homogenen festen und flüssigen Stoffe nur Regelmälsigkeiten vorhanden sind.

Wo aber für die Molekularvolumina der festen und homogenen flüssigen Stoffe regelmälsige Differenzen auftreten, sind diese Differenzen gleich denen für den gelösten Stoff. Dies ist für alle diejenigen Substanzen der Fall, bei denen angenommen werden darf, ${ }^{2}$ dal's das Molekulargewicht der homogenen Substanz nicht eine kom-

1 J. 'I'ratbe, Rer. deutsch. chom. Ges. (1892) 95, 2989.

${ }^{2}$ Vergl. weiter unten S. 333 , 
plexe Grölse ist. Nur wenn dieses der Fall ist, verschwindet die Regelmäfsigkeit in den Differenzen für die Molekularvolumina.

In allen bisher untersuchten Fällen wurde nun bei der Auflösung eines organischen Stoffes in Wasser eine Kontraktion festgestellt. Nehmen wir auch hier an, dals das Molekularvolumen eines Stoffes sich im Zustande der Lösung nicht ändert, ${ }^{1}$ so würde die Kontraktion auf Kosten des Wassers zu setzen sein, und wir würden zu einem Satze gelangen, nach welchem die Mehrzahl der organischen Stoffe auf das Wasser eine gleiche Anziehung ausübt.

Da nun für die eine scheinbare Ausnahme bildenden Stoffe eine Komplexität der flüssigen und festen Moleküle nachgewiesen wurde, so würde, bei Berücksichtigung dieses störenden Umstandes, auch hier jener Satz auf alle Moleküle ausgedehnt werden können.

Die Tabelle auf S. 325 lehrt ferner, dals für die verschiedensten organischen Säureionen die Kontraktion pro Gramm-Äquivalent gleich grofs (ca. $12.2 \mathrm{ccm}$ ) ist. Hieraus lälst sich schon mit einiger Wahrscheinlichkeit schliefsen, dals das, was für die verschiedenartigsten organischen Ionen gilt, auch für die verschiedenartigsten organischen nichtionisierten Moleküle gelten werde, denn dals die Addition des einen Wasserstoffatoms in dem Verhalten der Ionen eine Änderung herbeiführen sollte, ist sehr wenig wahrscheinlich.

Ich habe nun für eine gröfsere Anzahl organischer Stoffe in verdünnter wässeriger Lösung das molekulare Lösungsvolumen berechnet.

In folgender Tabelle findet sich unter $m$ das Molekulargewicht der Substanz. $V_{m}$ bezeichnet das aus Werten von Penkrs ${ }^{2}$ sowie Angaben von Beilstein, Landolt-Börnstejess Tabellen und LanDotT-Graham-Ottos Lehrbuch von Horstmann berechnete Molekularvolumen; $v_{m}$ das molekulare Lösungsvolumen für die verdünnte wässerige Lösung vom Prozentgehalt $p . \quad V_{m}$ und $v_{m}$ beziehen sich fast immer auf $15^{\circ} . \quad v_{m}$ ändert sich für verdünnte Lösungen mit dem Prozentgehalt meist gar nicht oder sehr wenig. In einigen Fällen (beispielsweise Alkoholen) findet eine kleine Zunahme statt, hier wurde $p$ so gewählt, dafs ${ }_{m}$ ein Minimum wurde.

Die Differenz $V_{m}-v_{m}$ bezeichnet die Kontraktion des Wassers

1 Vergl. S. 325.

${ }^{2}$ Perkin, Journ. pr. Chem. N. F. (1885) 31, 481. 
pro Gramm-Molekül gelösten Stoffes unter der Voraussetzung, dafs sein Molekularvolumen bei dem Lösungsvorgange ungeändert bleibt. Über $x$ vergl. weiter unten.

\begin{tabular}{|c|c|c|c|c|c|c|c|}
\hline & $m$ & $V_{m}$ & $v_{m b}$ & $p$ & $V_{m}-v_{m}$ & $x$ & $\begin{array}{l}x \text { korr. } \\
\text { bei } 20^{\circ}\end{array}$ \\
\hline Äthylenhydrat & 62.04 & 55.6 & 54.2 & 1.5 & 1.4 & 2.9 & $\cdots$ \\
\hline Methylalkohol & 32.03 & 40.2 & 37.2 & 30 & 3.0 & 3.4 & 2.32 \\
\hline Ameisensäure & 46.01 & 37.5 & 34.5 & 1 & 3.0 & 3.6 & - \\
\hline Allylalkohol & 58.04 & 67.7 & 63.2 & 5.8 & 4.5 & 1.9 & - \\
\hline Äthylalkohol & 46.04 & 57.9 & 52.7 & 20 & 5.2 & 2.7 & 1.65 \\
\hline Propylalkohol & 60.06 & 74.2 & 68.9 & 10 & 5.3 & 2.3 & $\ldots$ \\
\hline Essigsäure & 60.08 & 56.8 & 51.0 & 1 & 5.8 & 3.6 & 2.13 \\
\hline Isopropylalkohol & 60.06 & 75.9 & 69.7 & 6 & 6.2 & 2.9 & -- \\
\hline Pyridin & 79.07 & 82.8 & 76.6 & 10 & 6.2 & 0.9 & - \\
\hline Isoamylalkohol & 88.08 & 108.1 & 101.4 & 2.2 & 6.7 & 2.0 & $\ldots$ \\
\hline Propionsäure & 74.04 & 74.2 & 67.4 & 10 & 6.8 & 1.8 & $\ldots$ \\
\hline Isobutylalkohol & 74.07 & 91.8 & 84.7 & 7.4 & 7.1 & 1.9 & - \\
\hline Buttersänre & 88.06 & 91.1 & 83.7 & 10 & 7.4 & 1.6 & -- \\
\hline Aceton & 58.04 & 72.9 & 65.4 & 5.8 & 7.5 & 1.3 & $\ldots$ \\
\hline Methylacetat & 74.04 & 78.8 & 71.2 & 1 & 7.6 & 1.0 & - \\
\hline Äthylsulf hydrat & 62.1 & 74.5 & 66.8 & 0.2 & 7.7 & 1.0 & - \\
\hline Crotonsäure & 86.04 & 85.0 & 77.2 & 4 & 7.8 & $\ldots$ & -- \\
\hline Propylformiat & 88.06 & 96.8 & 88.6 & 2.2 & 8.2 & 1.0 & ... \\
\hline Methylpropionat & 88.06 & 95.7 & 87.1 & 4.4 & 8.6 & 1.0 & $\ldots$ \\
\hline Isobuttersäure & 88.06 & 92.2 & 83.6 & 10 & 8.6 & 1.4 & --- \\
\hline Dimethyläthylkarbinol & 88.06 & 108.1 & 199.4 & 4.4 & 8.7 & - & -..- \\
\hline Isovaleriansă̈ure & 102.07 & 109.3 & 00.0 & $\tilde{\mathbf{0}}$ & 9.3 & 1.4 & - \\
\hline Ätliylacetat & 88.06 & 97.1 & 187.7 & 4.4 & 9.4 & 1.0 & $\ldots$ \\
\hline Propylacetat & 102.07 & 114.3 & 04.9 & 1.3 & 9.4 & 1.0 & - \\
\hline Acctessigsaures Äthyl & 130.07 & 126.8 & 117.4 & 1 & 9.4 & 1.0 & - \\
\hline Athylpropionat & $102.07 !$ & 113.9 & 104.2 & 1.3 & 9.7 & 0.9 & - \\
\hline Paraldehyd & 132.08 & 132.3 & 122.2 & 6.6 & 10.1 & 0.9 & - \\
\hline Allylacetat & 100.06 & 108.6 & 97.8 & 1.3 & 10.8 & - & -- \\
\hline Nikotin & 162.1 & 160.3 & 149.9 & 9 & 10.4 & $\longrightarrow$ & -- \\
\hline Propylamin & 59.10 & 82.2 & 70.9 & 1.5 & 11.3 & - & $\ldots .$. \\
\hline Methylpropylketon & 86.07 & 106.2 & 94.3 & 1 & 11.9 & 1.1 & - \\
\hline
\end{tabular}

Die Werte $V_{m}-v_{n}$, welche unter der erwähnten Annahme die Kontraktion des Wassers pro Gramm-Molekül gelösten Stoffes bezeichnen, sind, wie man erkennt, sehr verschieden grofs, aber man sieht, dals dieselben in der That nach der Zahl 12 hin konvergieren.

Es ist nun aus verschiedenen, namentlich von Guxe aufgestellten Gleichungen kritischer Gröfsen, aus Messungen der Verdampfungswärmen und vor allem aus den von Ramsay und SHieuds ${ }^{1}$

${ }^{1}$ Ramsay und Scuneds, Zeitschr. phys. Chem. (1893) 12, 433. Ramsay und Aston, Zeitsehr. phys. Chem. (1894) 15, 89 und 98. Ramsay, Zeitschr. phys. 
untersuchten Beziehungen der Oberflächenenergie zur Temperatur übereinstimmend gefolgert worden, dafs ein Teil der homogenen Flüssigkeiten zu den nicht assoziierenden gehört, d. h. eine molekulare Grölse hat, die derjenigen im Gaszustande entspricht, ein anderer Teil dagegen als ,assoziierend" bezeichnet werden mufs, da das flüssige Molekül mehr oder weniger gröfser ist als das gasförmige.

Der Assoziationsfaktor würde diejenige Zahl sein, welche angiebt, um wie viel das Molekulargewicht im flüssigen Zustande grölser ist als für den gasförmigen Zustand.

Die Grölse dieser nach den verschiedenen Methoden berechneten Assoziationsfaktoren ist zwar noch recht verschieden, aber von Bedeutung ist es, dafs die Gruppierung der einzelnen Stoffe in den beiden Klassen, trotz der Verschiedenheit der Methoden, eine sehr übereinstimmende ist.

Unter $x$ finden sich nun die von Ramsay und SHIELDS berechneten Assoziationsfaktoren. Diese Faktoren bedürfen sicherlich vielfach der Korrektion; so berechnen die Verf. neuerdings die unter $x$ korr. berechneten Werte.

Bedenken wir auch, dals die T'emperaturen, für welche $x$ bestimmt wurde, vielfach andere sind, als die, für welche $V_{m}$ und $v_{m}$ berechnet wurden, so ist unverkembar, dal's im grofsen und ganzen die Werte $V_{n}-v_{i n}$ und $x$ einander parallel gehen.

Bei den nicht assozierenden Flüssigkeiten ist die Kontraktion meist gröfser als 8 und kleiner als 12 , bei den assoziierenden Flüssigkeiten dagegen sind die Werte oft ganz erheblich kleiner.

Die Assoziation der Moleküle bewirkt daher eine Volumverkleinerung, ${ }^{1}$ ebenso wie die Verbindung der Ionen mit dem Wasser. In hohem Grade wird demgemäls die Annahme wahrscheinlich, dafs bei Berücksichtigung der Assoziation jedes Gramm-Molekül eines nicht ionisierten Stoffes eine Kontraktion des Wassers von ca. $12.2 \mathrm{ccm}$ hervorbringt.

Einwertige Ionen, sowie nicht ionisierte Moleküle nichtleitender Stoffe scheinen demnach das Wasser um gleich viel zu kontrahieren. Anders scheint dies dagegen bei den mehrwertigen Ionen zu sein.

Chem. (1894) 15, 106. VAN ber WaAs, Zeitselur. phys. Chem. (1894) 13, 714. Ramsax und Aston, Journ. chem. Soe. (1894), 173. Ref. Zeitsehr: phys. Chem. (1894) 14, 572.

1 Fiir die Doppelsalzbildung gilt auffallenderweise das umgekehrte. Vergl. J. 'Traube, Dieve Katschr. (1895) 8, 74. 
Schon in einer früheren Abhandlung ${ }^{1}$ wies ich darauf hin, dafs die Abrahme der molekularen Lösungsvolumen mit wachsender Verdünnung für gleiche Konzentrationsintervalle pro Gramm-Aquivalent eines ternären Salzes erheblich grölser sei als für ein binäres Salz.

Wenn schon diese Thatsache eine grölsere Kontraktion für ein zwei- und mehrwertiges Ion wahrscheinlich macht, so scheint auch das Verhalten der mehrbasischen Säuren in der Tabelle S. 325 darauf hinzudeuten, dals hier die Kontraktion pro Gramm- ̈̈quivalent eines mehrwertigen Ions $=12.2$ beträgt.

Wenn $k$ die Anzahl Ionenäquivalente sind, in welche ein Molekül zufällt, und $\gamma$ der aus dem Leitvermögen abgeleitete Dissoziationsgrad, so würde hiernach die Kontraktion pro GrammMolekül eines beliebigen ionisierten oder nicht ionisierten Stoffes durch die Formel $c=12.2[1+(k-1) \gamma]$ gegeben sein.

Ebenso wird sich das von dem Einflusse der Assoziation befreite Molekularvolumen eines beliebigen Stoffes berechnen müssen, wemn man dem molekularen Lösungsvolumen für verdünnte Lösungen obigen Wert $c$ hinzuaddiert.

Nun noch einige Worte über den Zusammenhang, welcher anscheinend zwischen Kontraktions- und elektrischen Erscheinungen besteht.

Für die Volumerscheinungen gilt der Satz:

Gleiche Äquivalente verschiedener Ionen bringen bei der Lösung in Wasser die gleiche Volumverminderung $(=12.2 \mathrm{ccm})^{2}$ hervor.

Das Faraduysche Gesetz lautet:

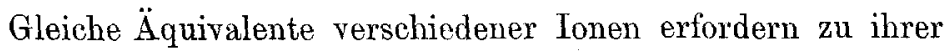
Abscheidung aus der Lösung die Aufwendung gleicher Elektrizitätsmengen (96540 Coulombs).

Die Anhänger der elektrolytischen Dissoziationstheorie nehmen an, dals die Ionen im Wasser elektrisch geladen seien. Drude und NERNst betrachten die elektrische Ladung der Ionen als die Ursache der Volumkontraktion. Mir scheint die Analogie der obigen beiden Gesetze darauf hinzudeuten, dals die Ionen in Lösung überhaupt nicht elektrisch geladen sind, dafs dieselben erst elektrisch

1 J. Traube, Ber. deutsch. chem. Ges. (1892) 25, 2530.

2 Der Zahl $12.2 \mathrm{ccm}$ entspricht ein l)ruck vou ca. 250 Atmosphären uml eine Würmemenge vou $\frac{1}{2} \cdot 165 \mathrm{~K}$. 
geladen werden im Moment ihrer Abscheidung, und dals die elektrische Ladung der Ionen demnach nicht die Ursache, sondern die Folge der Volumkontraktion sein werde. Raumenergie würde sich in elektrische verwandeln, und das Gesetz von Faraday wäre die Folge des hier aufgestellten Satzes.

Allerdings handelt es sich hier zunächst nur um eine Analogie und noch nicht um den Nachweis eines quantitativen Zusammenhanges beider Gesetze, welcher vorläufig noch mit Schwierigkeiten verbunden war.

Charakteristisch für die nahen Beziehungen, welche anscheinend zwischen elektrischen und Volumerscheinungen bestehen, ist die Stellung, welche das Wasser inmitten der verschiedenen Lösungsmittel eimimmt.

Während nämlich die Kontraktion pro Gramm-Molekül für Wasser = ca. 12,2 ccm betrug, werden zwar für Lösungen von Nichtleitern in organischen Tösungsmitteln, insbesondere Flüssigkeitsgemischen, auch Volumänderungen beobachtet, aber diese Volumänderungen sind im Vergleich zum Wasser meist höchst unbedeutend. Alles scheint dafür zu sprechen, dal's hier die kleinen Volumänderungen ausschliefslich durch Assoziationen und Dissoziationen komplexer Molekïle bedingt werden.

Erinnert man sich nun daran, dal's selbst wässerige Lösungen von Nichtleitern die Elektrizität unendlich viel besser leiten als die meisten Gemische organischer Flüssigkeiten, für welche vielfach nur eine metallische Leitung angenommen wird, so fällt anch hier die Analogie elektrischer und räumlicher Erscheinungen auf. ${ }^{1}$

Zunächst sehr auffallend verhielten sich Lösungen von Elektrolyten in Äthylalkohol, und insbesondere Methylalkohol. Hier finden, wie aus demnächst zu veröffentlichenden Zahlen folgt, allgemein Kontraktionen statt, welche weit erheblicher sind als für Wasser.

Aber von Schald ${ }^{2}$ wurde für Lösungen von Salzsäure in Methylalkohol, und von mir für Jodnatrium in Methylalkohol nachgewiesen, dals diesen Lösungen auch ein unerwartet grolses, sich mit der Konzentration stark änderndes Leitvermögen zukommt.

1 So wurde beispielsweise in Alkoholwassergemischen für dieselbe mittlere Konzentration ein Minimum von mol. Lösungsvolumen und elektrischer Leit.fähigkeit festgestellt.

2 Somati, Zeitsehr. physs. Chem. (1894) 14, 701. 
Also auch hier scheint ein Zusammenhang beider Arten von Erscheinungen zu bestehen.

Hinweisen möchte ich endlich auf eine $u$. a. von TammaN ${ }^{1}$ aufgestellte Beziehung zwischen Binnendruck, Kontraktion und Kompressionskoëffizienten.

Nimmt man an, dals bei der Neutralisation äquivalenter wässeriger Lösungen von Säure und Basis die Änderung des Binnendruckes gleich ist der Volumänderung, dividiert durch den Kompressionskoëffizienten der Salzlösung, so berechnen sich aus den von TAMman abgeleiteten Werten für Binnendruck sowie Kompressionskoëffizienten als Werte der Volumdilatation pro $0.5 \mathrm{Mol}$. $\mathrm{NaCl}$, $\mathrm{KCl}, \mathrm{NaBr}$ und $\mathrm{KBr}$, die Werte $\Delta v=11.3,11.0,9.6$ und $11.7 \mathrm{ccm}$. Gefunden wurden 9.8, 10.0, 10.0 und $10.2 \mathrm{ccm}$.

Tamman glaubt an eine nur zufällige Übereinstimmung, indem er annimmt, dals die Volumänderung der Änderung der Binnendrucke plus der Volumänderung entspricht, welche bei der Bildung eines Gramm-Moleküls Wasser aus seinen Ionen $\mathrm{H}$ und $\mathrm{OH}$ resultiert.

Diese letztere Volumänderung ist aber nach unserer Annahme $=0$, womit die in Anbetracht der nicht völligen Dissoziation vorzügliche Übereinstimmung von Beobachtung und Berechnung in gutem Einklang steht. ${ }^{2}$

1 Tamman, Zeitsehr. phys. Chem. (1803) 11, 691. Vergl. auch Ruppin, Zeitschr. phys. Chem. (1894), 14, 484.

${ }^{2}$ Vergl. übrigens die soeben Zeitschr. phys. Chem. (1895) 16, 139 ersehienene Abbandlung von Tamman. Verf. kommt hier zu interessanten Schlüssen, die sich mit denen von mir in Einklang bringen lassen.

Organ. Laboratorium der Techn. Hochschule Berlin, den 18. Februar 1895.

Bei der Redaktion eingegangen am 20. Februar 1895. 